\title{
COPD-Patienten haben es oft auch am Herzen
}

\begin{abstract}
Komorbiditäten wie Diabetes oder psychiatrische Erkrankungen sind bei COPD-Patienten keine Seltenheit. Mit weitem Abstand aber überwiegen begleitende kardiovaskuläre Erkrankungen. Prof. Dr. med. Heinrich Worth, Fürth, rät deshalb dringend, bei COPD nicht nur die Lunge, sondern auch das Herz im Auge zu behalten.
\end{abstract}

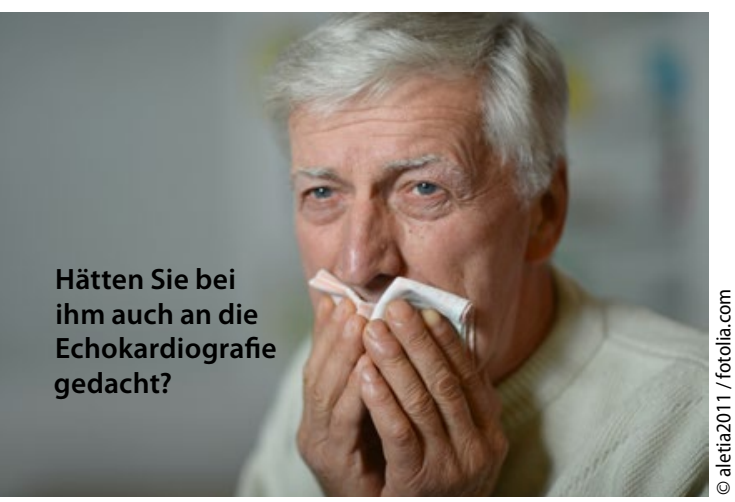

Aus dem DACCORD-Register mit über 12.000 Patienten geht hervor, dass bei mehr als der Hälfte der ambulanten COPD-Patienten auch eine koronare Herzkrankheit, eine Herzinsuffizienz oder arterielle Hypertonie vorliegt. Von den schwer kranken COPD-Patienten in der TORCH-Studie verstarb jeder vierte an einer Herz-Kreislauf-Erkrankung.

Die Problematik der Konstellation COPD plus kardiovaskuläre Erkrankungen zeigt sich auch aus anderer Perspektive: So haben Patienten mit Herzinsuffizienz oder ischämischer Herzerkrankung eine deutlich schlechtere Prognose, wenn noch eine COPD hinzukommt. Häufig besteht in Verbindung mit einer COPD auch eine eingeschränkte links-

Tab. 1 Auswirkungen der COPD auf die Herzfunktion

\begin{tabular}{|c|c|c|c|c|}
\hline \multicolumn{2}{|c|}{ Ruhe } & Belastung & \multicolumn{2}{|l|}{ Kardiale Auswirkung } \\
\hline$\uparrow$ & Überblähung & $\uparrow \uparrow \uparrow$ & LV-Funktion & $\downarrow$ \\
\hline$\uparrow$ & Atemarbeit & $\uparrow \uparrow \uparrow$ & Herzzeitvolumen & $\uparrow$ \\
\hline$-/ \uparrow$ & intrathorakaler Druck & $\uparrow \uparrow \uparrow$ & venöser Rückstrom & $\downarrow$ \\
\hline$-/ \uparrow$ & pulmonale Hypertonie & $\uparrow \uparrow \uparrow$ & $\begin{array}{l}\text { Herzzeitvolumen } \\
\text { Rechtsherzbelastung }\end{array}$ & \\
\hline
\end{tabular}

ventrikuläre Funktion, die sich ungünstig auf das Überleben auswirkt, aber vielfach unentdeckt bleibt.

\section{COPD, eine systemische Erkrankung}

Die COPD wird als inflammatorische Krankheit angesehen. Diese Entzündung macht nicht in der Lunge halt, sondern erreicht über das Endothel auch andere Organe wie die Herzkranzgefäße. Worth:. „Wir haben bei der COPD eine Erkrankung mit systemischen Auswirkungen auf Herz, Gefäße und vermehrt Krankheiten wie Diabetes, metabolisches Syndrom."

Außerdem beeinträchtigen die Veränderungen in der Lunge die Funktion des Herzens mechanisch (siehe Tab. 1). Ein Teil der Patienten leidet auch unter einer pulmonalen Hypertonie, die das rechte Herz belastet und zum Cor pulmonale beiträgt.

\section{Betablocker senken COPD- Sterblichkeit}

Jensen MT et al (ERJ 2012) stellten fest, dass mit dem Schweregrad der COPD die RuheHerzfrequenz ansteigt und dass dies die Lebenserwartung verkürzt. In der Kardiologie werden gegenwärtig Frequenzen um die 60 Schläge/Minuten angestrebt. Von diesem Zielwert sind Patienten mit schwerer COPD weit entfernt, erklärte Worth. Zu hohe Werte sollte man deshalb durchaus auch beim Patienten mit einer chronisch obstruktiven Bronchitis senken, etwa mit ei- nem kardioselektiven Betablocker oder mit dem If-Kanalblocker Ivabradin.

Betablocker sind in der Therapie der obstruktiven Atemwegserkrankung umstritten, weil sie eine Obstruktion auslösen oder verstärken können. Für Asthmatiker werden sie deshalb auch nicht empfohlen. Das gilt aber nicht für die COPD. Hier können sich Betablocker, insbesondere nach Herzinfarkt oder bei Herzinsuffizienz, als hilfreich erweisen. Ein Problem sieht der Pneumologe eher darin, dass eine COPD viele Ärzte davon abhält, bei Herzinsuffizienz Betablocker einzusetzen. Dies geht auch aus dem DACCORD-Register hervor. Die Wahl sollte allerdings auf kardioselektive Betablocker fallen, weil damit weniger unerwünschte Effekte auf die Atemwegsobstruktion zu erwarten sind. Außerdem sollte man darauf achten, dass die Herzfrequenz unter dieser Therapie wirklich abnimmt. Einer Studie aus Schottland (Short PM et al. BMJ 2011;342:2549) zufolge hat die Gesamtmortalität bei COPD-Patienten bei Gabe eines Betablockers bei einem mittleren Follow-up von 4,35 Jahren um 22\% abgenommen im Vergleich zu Patienten ohne Betablocker. Für diesen positiven Effekt spielte die Art der gleichzeitigen COPDTherapie keine Rolle.

\section{Bei COPD Lungenfunktion und Echo}

Worth zufolge sollte man einem COPDPatienten mit kardialer Ischämie den Betablocker auch deshalb nicht vorenthalten, weil ähnlich wie bei der Herzinsuffizienz die Sympathikusaktivität erhöht ist. Betablocker reduzieren die Sympathikusaktivität und verbessern damit die Mortalität.

Generell sollte man, so Worth abschließend, bei COPD-Patienten an die Möglichkeit einer kardiovaskulären Komorbidität denken und deshalb nicht nur die Lungenfunktion bestimmen, sondern auch eine Echokardiografie durchführen.

- Martin Bischoff

- BDI-Symposium „Pneumologie 2015 - Was ist neu?" anlässlich des 121. Internistenkongresses am 19. April 2015 in Mannheim 